\title{
Neuronavigation as a Tool for Pre-Surgical Planning in Refractory Epilepsy Surgery
}

\author{
Valencia-Calderón $\mathbf{C}^{\mathbf{1}^{*}}$, Calderón-Valdiviezo $\mathrm{A}^{\mathbf{2}}$, Alvarez-Fernandez $\mathrm{B}^{\mathbf{1}}$, Rial-Basalo JC ${ }^{\mathbf{1}}$, Bernal-Carrillo $\mathrm{R}^{3}$ \\ ${ }^{1}$ Department of Neurosurgery, Central University Hospital of Oviedo, Oviedo-Spain \\ ${ }^{2}$ Primary Health Care Center "Antón Borja”, Rubí, Barcelona-Spain \\ ${ }^{3}$ Department of Neurosurgery, Pediatric Hospital "Baca Ortiz", Quito, Ecuador. \\ * Corresponding Author: Carlos Valencia Calderón, MD, PhD, Department of Neurosurgery, Central University Hospital of Oviedo, \\ Oviedo-Spain. E-Mail: carlos.valencia@sen.es
}

Received date: October 03, 2019; Accepted date: October 18, 2019; Published date: October 24,2019 Citation: Valencia-Calderón C, Calderón-Valdiviezo A, Alvarez-Fernandez B, Rial-Basalo JC, Bernal-Carrillo R, (2019) Neuronavigation as a tool for Pre-surgical Planning in Refractory Epilepsy Surgery. J. Neuroscience and Neurological Surgery.5(1);DOI:10.31579/2578-8868/095

Copyright:@ 2019 Carlos Valencia Calderón, This is an open-access article distributed under the terms of the Creative Commons Attribution License, which permits unrestricted use, distribution, and reproduction in any medium, provided the original author and source are credited.

Summary
Epilepsy is one of the most frequent chronic neurological pathologies, with high incidence and prevalence worldwide. A third
of these patients are resistant to treatment, which is known as refractory epilepsy. Most of these patients suffer epilepsy
secondary to epileptogenic lesions, where the surgery is the only treatment that could cure epilepsy. The goal of epilepsy surgery
is to remove the epileptogenic area with preservation of eloquent areas, and here the surgical experience, the neuroimaging
technology and the availability of image-guided surgery systems known as a neuronavigator plays a key role.
Objectives: To demonstrate the usefulness of neuronavigation in pre-surgical planning and in the surgery of refractory
epilepsy.
Method: A cross-sectional and analytical descriptive study was conducted based on 47 surgeries performed $(12$ resective, 32
palliative and 3 diagnostic) in patients with refractory epilepsy and mean age of 9.93 years (SD 4.1$)$. In 27 patients $(57.44 \%)$
the neuronavigator was used. In the group of patients operated with neuronavigation, the surgical time decreased by 47.17
minutes ( $=0.022)$, the amount of bleeding by 111.41 milliliters ( $p=0.011$ ) and the days of hospitalization by 6.68 days (p $=$
$0.005)$ compared with the group operated without neuronavigation. Complications in the neuronavigation group were $29.63 \%$
compared to $65 \%$ in the group operated without neuronavigation ( $\mathrm{p}=0.034$ ).
Conclusions: In our series, the use of the neuronavigator in the planning and development of the surgery had a significant
impact by reducing the amount of bleeding lost, the surgical time, the days of hospitalization, and the post-surgical
complications.

\section{Introduction}

Epilepsy is one of the most frequent chronic neurological pathologies, with an incidence of $50 / 100,000 /$ year and a prevalence between 0.5 and $2 \%$ worldwide [1,2]. A third of these patients are resistant to treatment with antiepileptic drugs [3]. ILAE defines refractory epilepsy as "one in which there has been a failure to 2 trials of antiepileptic drugs (FAE), in monotherapy or in combination, tolerated, appropriately chosen and appropriately used, to achieve the sustained absence of crisis" $[4,5]$.

Most of these patients suffer from focal epilepsy secondary to epileptogenic lesions, increasingly evidenced in direct correlation with the new brain neuroimaging techniques [6]. Epilepsy surgery is the only treatment that could cure epilepsy in patients with refractory epilepsy. This option should be considered as soon as drug resistance is evident, as several studies have shown that patients with active epilepsy have a mortality three times higher than the general population (7). In well- selected patients, epilepsy surgery is highly effective, obtaining freedom of crisis of up to $84 \%$, depending on the experience of surgeons and the technology available in their centers (8). The aim of epilepsy surgery is to remove the epileptogenic area with preservation of eloquent areas, and it is here that surgical experience and neuroimaging technology play a key role [9].

Images in the Preoperative Evaluation of Refractory Epilepsy.

The basic protocols for the acquisition of images in epilepsy were defined by the International League against epilepsy (ILAE) [4]. In the last 20 years there has been an important technological development in the area of epilepsy imaging, especially in the field of nuclear magnetic resonance, such as improvements in the equipment of 3 teslas and even 7 teslas, which have revolutionized the approach of these patients finding injuries that 
went unnoticed with 1.5 tesla equipment [10]. Magnetic resonance, both structural and functional, is the imaging modality of choice to determine and delimit the epileptogenic zone, as well as to define the surrounding structures.

These studies obtained in DICOM format allow the analysis of the images in the workstations of the neuronavigation teams.

\section{Neuronavigation}

In the last two decades, the evolution of neuroimaging techniques, both structural, functional and neuronavigation, have proven useful in presurgical planning of refractory epilepsy [11]. Neuronavigation consist of a sophisticated computer technology concerning software and hardware, that processes digitized neurorradiological images in DICOM format (process called planning), interacting and matching point by point imaging data with the real anatomical structures of the patient obtaining a neuroanatomic imaging map in $3 \mathrm{D}$ through a process called registration. Accuracy and safety when locating the lesion, choosing the best route or path to address it without damaging adjacent tissues, reducing surgical instrumentation time and radical removal of the lesion are some of the advantages of this technique.[11].

Stereotactic location is a neurosurgical technique capable of achieving the location and approach of any intracranial point or target through a system of $\mathrm{x}, \mathrm{y}, \mathrm{z}$ coordinates, through a device that is attached to the patient's head and guided by tomography images Axial computational (CT), magnetic resonance image (MRI), angiography, or positron emission tomography (PET).

The main disadvantage of navigation systems is the well-known brain shift or cerebral movement that is caused by cerebral edema, secondary to cerebral decompression or after cerebrospinal fluid drainage, which produces a calculation error due to this displacement, which can be corrected with the acquisition of intraoperative images of Ultrasound, CT or MRI, with systems that are compatible with neuronavigation equipment, which would allow visualizing changes in real time [12].

For intraoperative use, neuronavigation systems must relate the physical location of a patient to the preoperative models through a transformation that relates the two paradigms through patient-to-image mapping [12]. The main components of any neuronavigation system are a location device which will track the position of the patient and the surgical tools, a computer console that will present the information of the acquired images and finally accessories such as navigation probes and Frameworks.

The use of neuronavigation in lesion refractory epilepsy has not yet been adequately standardized. The objective of this work is to demonstrate the usefulness of neuronavigation in the pre-surgical planning of refractory epilepsy based on the experience acquired in the five years of use of neuronavigation in epileptic patients in our center.

\section{Materials and Methods}

A descriptive cross-sectional and analytical study that reviewed the data of patients with refractory epilepsy who were operated in our hospital with or without neuronavigation was conducted. Our hospital is a national reference pediatric center located in a city with a population of 2,576,287 inhabitants, and 723,073 inhabitants under 15 years. Since 2013 the hospital has an epilepsy surgery program and a multidisciplinary committee made up of specialists in neurology, neurosurgery, neurorradiology, neuropsychology, nutrition and neuroendocrinology. During the committee meetings, compliance with the refractoriness criteria, the frequency of the crises, the impact on the quality of life in relation to the crises, the indication of the type of medical and / or surgical, curative or palliative treatment were verified. Likewise, family members were informed of the recommendations issued by the committee and informed consent was obtained.

All patients with refractory epilepsy discussed by the epilepsy committee between January 2013 and December 2016, in total 55 patients, who met the inclusion criteria were included.

Pre-surgical planning was reviewed through neuronavigation, medical records and operative protocols for all cases.

\section{Technology used MRI of the Brain:}

Brain MRI study conducted with a team of 3.0 Phillips brand teslas (Medical Systems, DA Best, The Netherlands), which followed an epilepsy protocol that included simple volumetric T1 and T1 sequences and contrasted contrast with axial, coronal and sagittal cuts of up to a minimum of $0.4 \mathrm{~mm}$ thick, T2 and FLAIR; images by diffusion tensor were obtained with a minimum of 16 addresses and were assigned by agreement the colors red, green and blue to the right-left, anterior-posterior and superiorinferior orientations respectively; multivoxel spectroscopy and arterial and venous angio-MRI in all cases. The images were acquired in DICOM format and subsequently transferred to a workstation where the threedimensional analysis of the study was performed, as well as the presurgical planning of each case.

\section{Neuronavigator:}

We use a neuronavigator consisting of:

a) a BrainLab Z800 workstation, with the iPlan version 3.0.5 program where images acquired on magnetic resonance were loaded. The trajectories that were to be followed during the surgery were planned and the paths of the cutaneous flap opening, as well as the craniotomy and durotomy were delimited. Objects of images of interest were created, both normal and structural injuries; Vascularization was reconstructed by angio-MRI studies, and tractography of fascicles of interest.

b) In the volumetric analysis we used the function called Automatic Segmentation, which uses a knowledge-based segmentation approach that is capable of identifying brain structures by comparing the patient's images with a set of images from an atlas included in the iPlan, which contains defined anatomical structures. The application finds the direct correspondence between the set of images of the patient and the set of images of the atlas and transfers all the delimited structures of the anatomical atlas to the images of the patient.

c) A function called Fiber tracking of the iPlan 3.0 program was used to study the tractography. This function is based on the measure of diffusion anisotropy in the brain calculated from images of the diffusion tensor (DTI) acquired in several directions. The diffusion direction of the water along the potential fibers of the white substance was calculated for the entire volume explored.

d) The information planned in BrainLab Z800 workstation was transferred to a BrainLab Curve system with dual screen, where the facial and cranial anatomy points of the patient already anesthetized and fixed in the craniostat were registered. Registration was made using an infrared camera and a pointer.

\section{Surgical Planning.}

Once the images were presented on the screen of the planning station of the neuronavigator, the lesion, epileptogenic zone, corpus callosum, tonsil, hippocampus were drawn and colored, as well as eloquent or vascular structures of importance. Then the three-dimensional reconstruction was performed, as well as, triplanar (sagittal and coronal sections).

Upon this reconstruction, the desired entry points, target, and trajectories were drawn, immediately visualizing some of the eloquent or vascular structures near the trajectories.

\section{Intraoperative Registration.}

Once in the operating room, the patients were placed in a surgical position, with head fixation with a 3-point head (craniostat) (in the hospital they have the Mayfield Modified Skull). Next, a reference star (not sterile) was fixed, on one side or the other depending on the distribution of the 
operating room and the craniotomy as well. The recording chamber was usually located at the patient's feet usually on the right side. The calibration was performed by moving the cameras until observing the 3 reflective spheres of the star and those of the pointer, by using the camera's own laser. And then the patient's facial and / or cephalic surface was recorded.

Registration was always carried out under non-sterile conditions; the patient's face was placed as far as possible in front of the camera. Through the facial recognition pointer, multiple points of the malar area, orbital flange, superciliary flange, and nose were acquired, since these structures were the least mobile. In this way, a facial scan was performed, which the neuronavigation system used to calculate and obtain a three-dimensional model of the patient's facial anatomy.

Then, this model, which represented the position of the patient's head already in the operating room, was correlated with the diagnostic radiological data, and the registration was completed. To verify the correct registration, the anatomical reference test was performed as a control of the accuracy of the system. The pointer was placed on nonmovable structures such as the nasion, tip of the nose and swallow.

Next, we proceeded to locate the point of entry, and delimitation of the cutaneous surface of the region to be resected, to define the limits of the surgical incision and craniotomy. Next, the non-sterile star was removed, aseptic and antiseptic, sterile star placement and sterile fields were performed. And the surgical procedure was continued, using the registration tips or calibrated instruments to be recognized by the navigation system.

\section{Source of data Collection}

For the evaluation of the cases, medical records data were used, as well as the external consultation and hospitalization files, as well as data of the patients that were selected by the epilepsy committee, all the imaging studies that were found in the neuronavigator database.

\section{Data Collection Instruments}

Data collection sheets created for specific research purposes were made, data were collected from the statistical archives, the medical records and photographic archives available from the patients who were recorded in the Neuronavigator of patients with refractory epilepsy that were discussed in the epilepsy committee of the Baca Ortiz Pediatric Hospital between January 2013 and December2016 and processed in the neurosurgical department of the Central University Hospital of Oviedo. Statistical analysis of the data: To describe the results of the study variables, descriptive statistics methods were used: for numerical variables the mean and standard deviation were used, for the categorical variables percentages were used. For the analysis of the relationship between variables, non-parametric statistics were used, specifically the comparison of dependent means of numerical variables (crisis frequency

and quality of life scale score) was performed using the Wilcoxon test, and the comparison of variables. categorical through the X2 test. Data analysis was performed with the SPSS (Statistical Package for the Social Sciences) version 22.0 (SPSS, Inc., Chicago, IL, USA). Ethical and legal aspects: Since this is a descriptive study and therefore without intervention, it did not require approval by a bioethics committee, although it was approved by the Department of Teaching and Research of the hospital. In collecting data, the physical and emotional integrity of each patient was preserved, as well as their confidentiality. This work has no conflicts of interest with the company BrainLab.

\section{Results}

During the years 2013 - 2016, 55 cases of patients with refractory epilepsy were presented in the "Multidisciplinary Committee of Epilepsy" (table 1). For the study, inclusion and exclusion criteria were applied (table 2), obtaining a sample of 47 cases ( 2 patients were excluded because they had lesions in eloquent areas and 6 requested voluntary discharge) (figure 1$3)$.

Of the 47 epilepsy surgeries, $57.44 \%$ (n 27) were performed with the use of neuronavigation (21 of them were performed in the last two years $77.78 \%$ ) (table 3-5).

When comparing the results of surgical time, bleeding during the surgical procedure and days of hospitalization obtained from surgeries performed with and without the use of neuronavigation, we observed that with the use of the neuronavigator, the surgical time decreased by 47.7 minutes $(\mathrm{p}=$ $0.022)$, bleeding in 111.41 milliliters $(\mathrm{p}=0.011)$ and days of hospitalization in 6.68 days $(\mathrm{p}=0.05)$ (table 6$)$.

The complications in the neuronavigation group were $29.63 \%$ (eight patients) compared with $65 \%$ in the group operated without neuronavigation $(\mathrm{p}=0.034)$ (table 7$)$. The complications described were surgical site infection, hemiplegia, transient hemiparesis. Without the use of neuronavigation, 13 patients presented complications $(65 \%)$. The complications described were motor deficits (hemiplegia, transient hemiparesis, mutism) (Figure 4).

The table 8 describes the absolute frequency of mortality in both groups, being higher in the group operated without neuronavigator. These two deaths were not related to the neuronavigation process (one death due to pneumonia and another due to severe hyponatremia).

\begin{tabular}{|c|l|}
\hline & Outcome \\
\hline Sample age & Mean 9.93 years (SD 4.1). Rank 1 to 18 years \\
\hline Sex & $\begin{array}{l}\text { Female: 24 (51\%) } \\
\text { Male: 23 (49\%) }\end{array}$ \\
\hline \multirow{5}{*}{ Etiology of epilepsy } & $\begin{array}{l}\text { Perinatal lesion: } 11(23.4 \%) \\
\text { Malformation of cortical development: } 12(25.53 \%) \\
\text { Post-infection: 8 (17.02\%) } \\
\text { Hypothalamic hamartoma: 2(4.26\%) } \\
\text { Rasmussen encephalitis: } 2(4.26 \%) \\
\text { Post-traumatic: 1 (2.13\%) } \\
\text { Hippocampal sclerosis: } 1(2.13 \%) \\
\text { Tumors: } 8 \text { (17.02\%) } \\
\text { Unidentified: 2 (4.26\%) }\end{array}$ \\
\hline
\end{tabular}

Table 1. Describes the variables of the patient and the etiology of epilepsy. 


\section{Inclusion criteria}

Children from 0 to 15 years old, or older with the approval of the Ministry of Public Health

Patients diagnosed with epilepsy refractory to medical treatment

Patients who have all protocol imaging studies in the epilepsy committee.

Table 2: shows the inclusion criteria of the patients who participated in this study.

\begin{tabular}{|r|c|c|c|}
\hline Year & Total epilepsy surgeries & $\begin{array}{c}\text { Epilepsy surgeries with } \\
\text { neuronavigator use }\end{array}$ & Percentage \\
\hline 2013 & 2 & 1 & 50 \\
\hline 2014 & 14 & 5 & 35.71 \\
\hline 2105 & 14 & 11 & 78.57 \\
\hline 2016 & 17 & 10 & 58.82 \\
\hline TOTAL & 47 & 27 & 57.44 \\
\hline
\end{tabular}

Table 3: Percentage of epilepsy surgeries with the use of neuronavigator.

\begin{tabular}{|c|c|c|c|}
\hline \multirow{2}{*}{ Type of Surgery } & \multicolumn{2}{|c|}{ Use of Neuronavigation } & \multirow{2}{*}{ Total } \\
\cline { 2 - 4 } & $\mathrm{Si}$ & $\mathrm{No}$ & 12 \\
\hline Resectives & 8 & 4 & 32 \\
\hline Palliatives & 17 & 15 & 3 \\
\hline Diagnostics & 2 & 20 & 47 \\
\hline Total & 27 & 1 & \\
\hline
\end{tabular}

Table 4: Type of surgery and use of neuronavigator, in epilepsy surgeries.

\begin{tabular}{|l|c|c|c|}
\hline \multirow{2}{*}{ Surgical procedure } & Use of Neuronavigation & \multirow{2}{*}{ Total } \\
\cline { 2 - 4 } & $\mathrm{Si}$ & $\mathrm{No}$ & \multirow{2}{*}{30} \\
\hline Callosotomy & 17 & 13 & 1 \\
\hline Callosotomy + Hamartoma surgery & 0 & 1 & 1 \\
\hline Cortical Resection & 1 & 0 & 2 \\
\hline Hemispherectomy & 1 & 1 & 2 \\
\hline Standard temporal lobectomy & 2 & 0 & 2 \\
\hline Temporal Anterior lobectomy & 1 & 1 & 2 \\
\hline Tumor surgery & 3 & 3 & 2 \\
\hline Electrode Mesh Placement & 0 & 1 & 2 \\
\hline Biopsy & 2 & 0 & 2 \\
\hline Total & 27 & 20 & 2 \\
\hline
\end{tabular}

Table 5: Surgical procedures performed with and without the use of neuronavigator, in epilepsy surgeries. Of the surgical procedures, 12 were resective, 32 were palliative, and 3 diagnostics. 


\begin{tabular}{|c|c|c|c|c|c|c|}
\hline \multirow{2}{*}{ Year } & \multicolumn{2}{|l|}{ Surgical time in minutes } & \multicolumn{2}{l|}{ Bleeding in milliliters } & \multicolumn{2}{l|}{ Si } \\
\cline { 2 - 7 } & No & $\mathrm{Si}$ & No & $\mathrm{Si}$ & $\mathrm{No}$ & 10 \\
\hline 2013 & 175 & 120 & 100 & 40 & 6 & 7,6 \\
\hline 2014 & 258,89 & 186 & 316,89 & 170 & 15,67 & 7,27 \\
\hline 2015 & 223,33 & 198,18 & 283,33 & 239,55 & 9 & 9 \\
\hline 2016 & 247,86 & 212,5 & 407,14 & 303 & 17,29 & 8,07 \\
\hline Total & 245,5 & 198,33 & 352,85 & 241,44 & 14,75 & 0,005 \\
\hline$p$ & \multicolumn{2}{|c|}{0,022} & & & & \\
\hline
\end{tabular}

Table 6: Post-surgical results according to neuronavigator use in epilepsy surgeries.

\begin{tabular}{|c|c|c|c|}
\hline \multirow{2}{*}{ Operating Complications } & \multicolumn{2}{|c|}{ Use of Neuronavigation } & Total \\
\cline { 2 - 4 } & $\mathrm{Si}$ & $\mathrm{No}$ & 6 \\
\hline Local infection & 4 & 2 & 3 \\
\hline Cerebrospinal fluid fistula & 1 & 2 & 3 \\
\hline Transitory mutism & 0 & 3 & 5 \\
\hline Transient hemiparesis & 1 & 4 & 1 \\
\hline Hemiplegia & 1 & 0 & 1 \\
\hline Hypothalamic Failure & 1 & 1 & 2 \\
\hline Pneumonia & 19 & 7 & 26 \\
\hline Any & 27 & 20 & 47 \\
\hline
\end{tabular}

Table 7: Type of post-surgical complications with and without the use of neuronavigation.

\begin{tabular}{|c|c|c|c|}
\hline \multirow{2}{*}{ Mortality } & \multicolumn{2}{|c|}{ Neuronavegation use } & Total \\
\cline { 2 - 4 } & Si & No & $2 *$ \\
\hline Death & 0 & 18 & 45 \\
\hline Alive & 27 & 20 & 47 \\
\hline Total & 27 & & \\
\hline
\end{tabular}

Table 8: Mortality with and without neuronavigation use. 


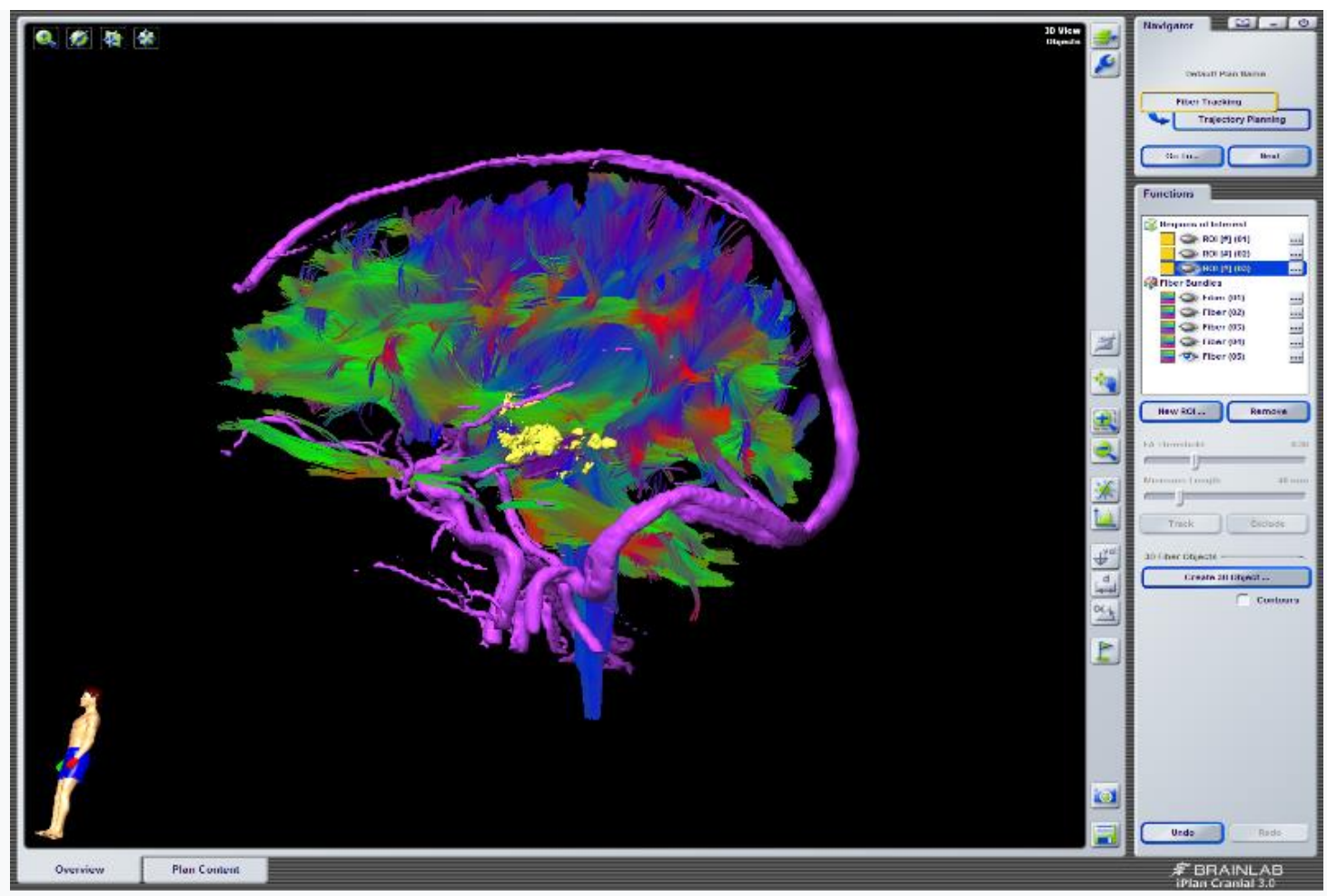

Figure 1: Six-year-old boy with temporal refractory epilepsy. MRI post-processing in which we have excluded the brain volume with 3D reconstruction of temporal cortical dysplasia (yellow) fused with tractography of the all brain and venous angio-MRI.

The study of this image has several connotations, such as the location in the space of this lesion, as well as its proximity to the vascular structures and its relationship with the language pathways.

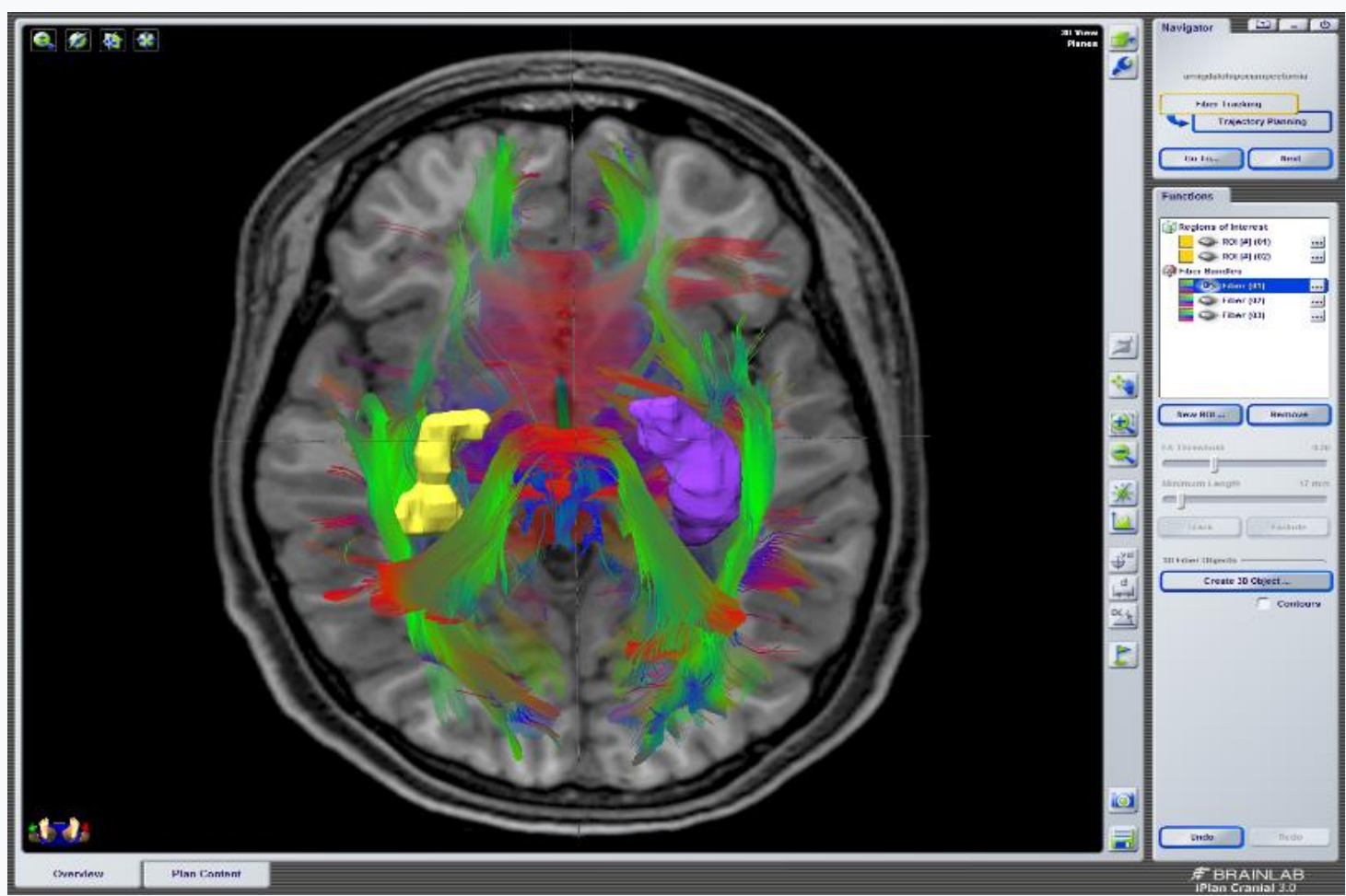

Figure 2A: Sixteen-year-old woman with right mesial temporal sclerosis. Three-Dimensional model built on a neuronavigation workstation. A). The illustration shows the affected hypocammpus (yellow) smaller that the left one (violet), segmented slice by slice on axial images. 


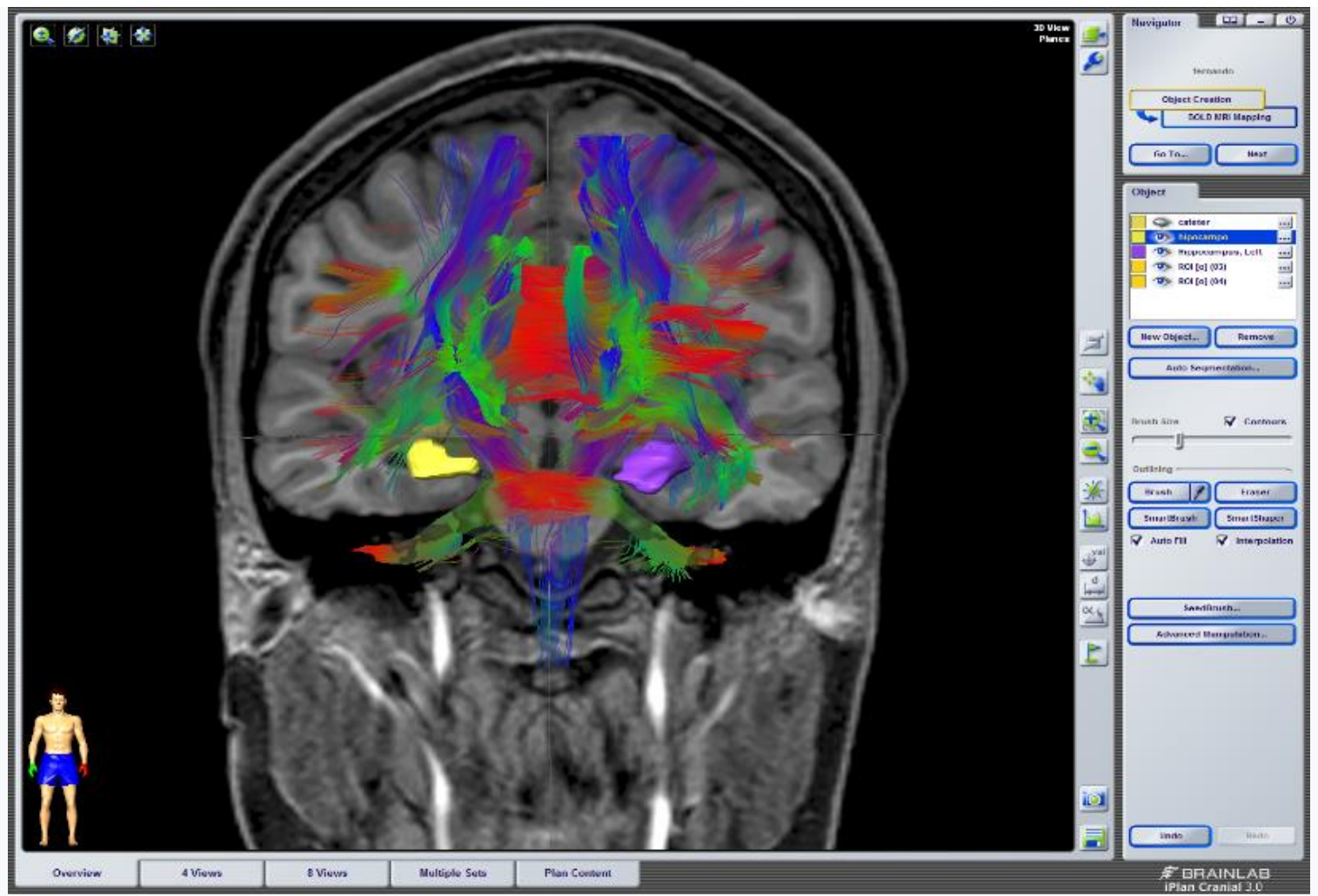

Figure 2B: The same patient of figure 2A. The coronal image allows comparing and observing the lower fiber density in the right hemisphere as a sign of suffering due to the high frequency of seizures.

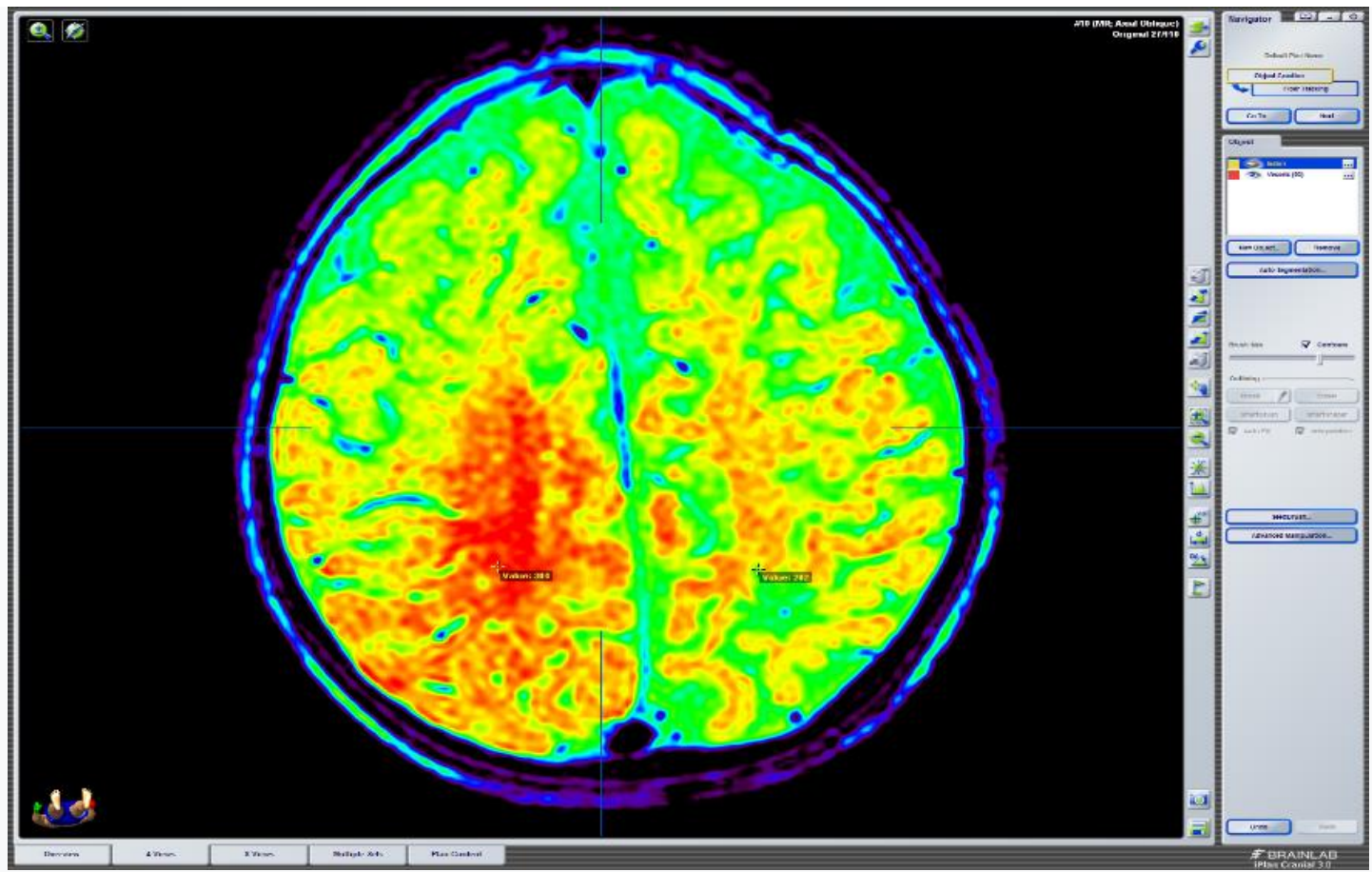

Figure 3A: Four-year-old boy with Lennox-Gastaut syndrome with an average of 160 seizures per month. The MRI showed an extensive dysplasic lesion at the right parietal and occipital lobes. This illustration called Hot-Metal let's see the size of the lesion. 


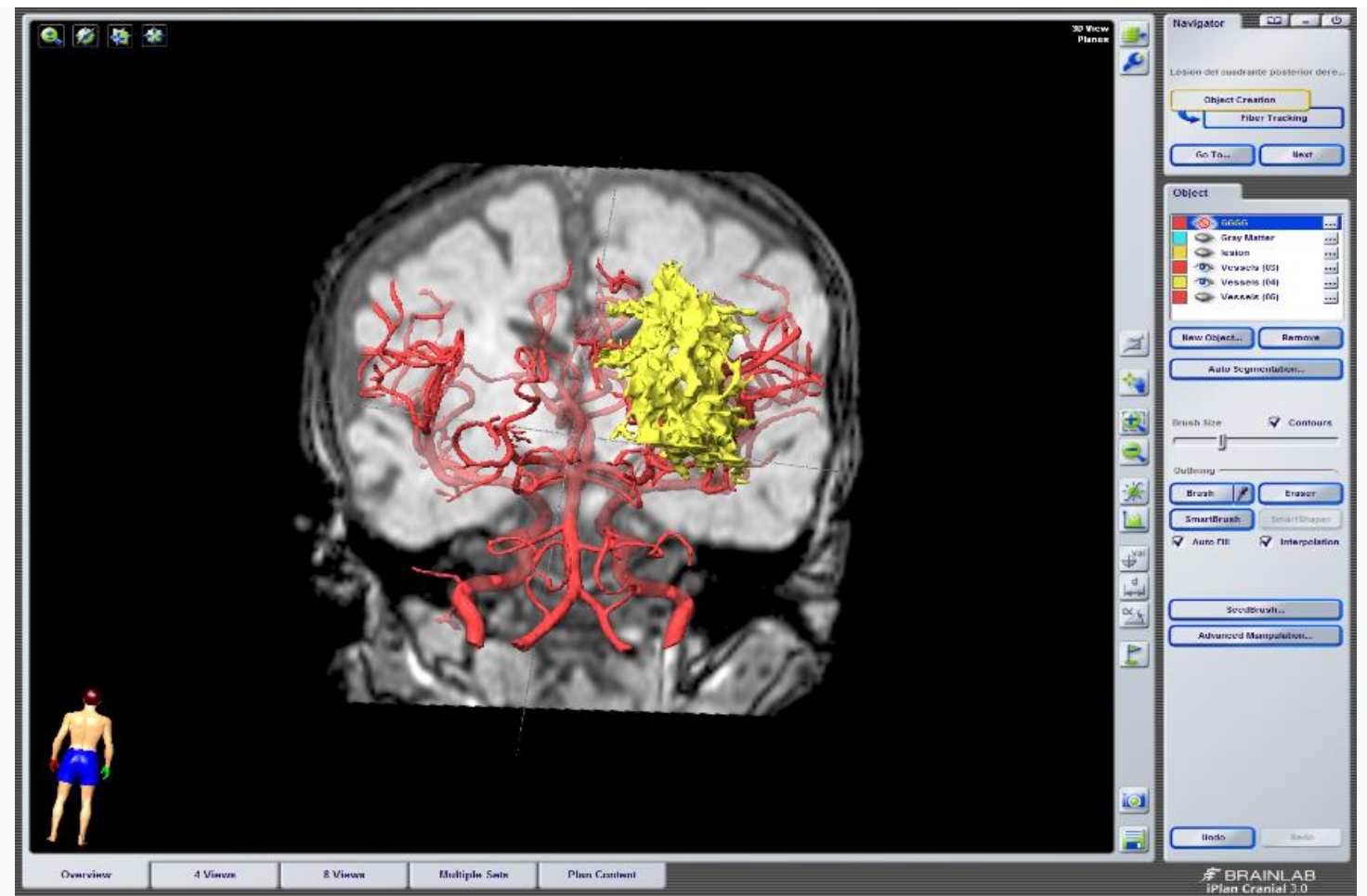

Figure 3B: The same patient of figure 3A. Three-dimensional reconstructions with and without the brain, show the volumetric lesion and its vascular relations with the Willis polygon. A right parieto-occipital quadrantectomy guided with electrocorticography was performed.

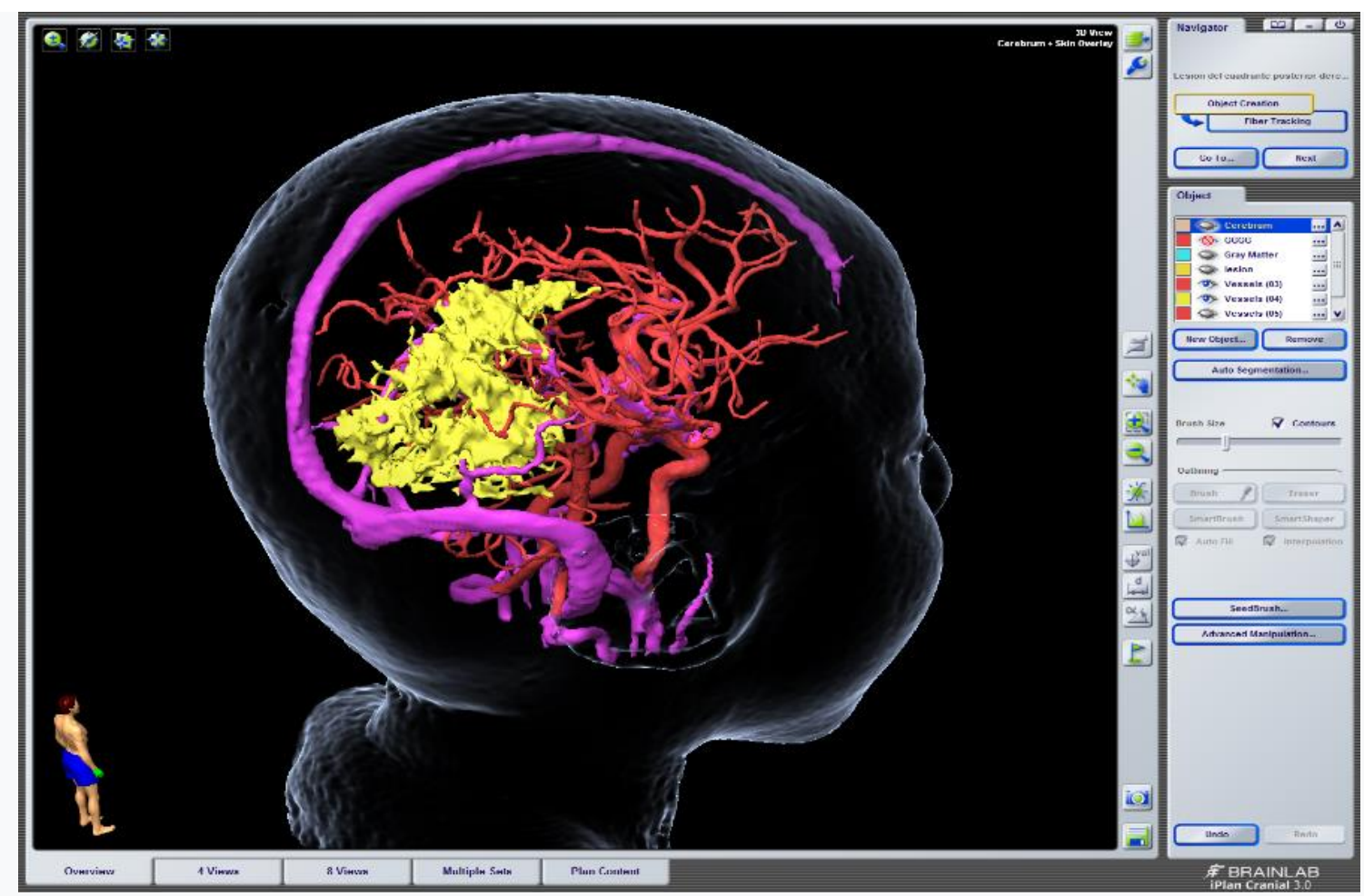

Figure 3C: The same patient of figure 3A. Three-dimensional reconstructions with and without the brain, show the volumetric lesion and its vascular relations with the venous system. After surgery the patient had between 4 and 6 seizures per month. 


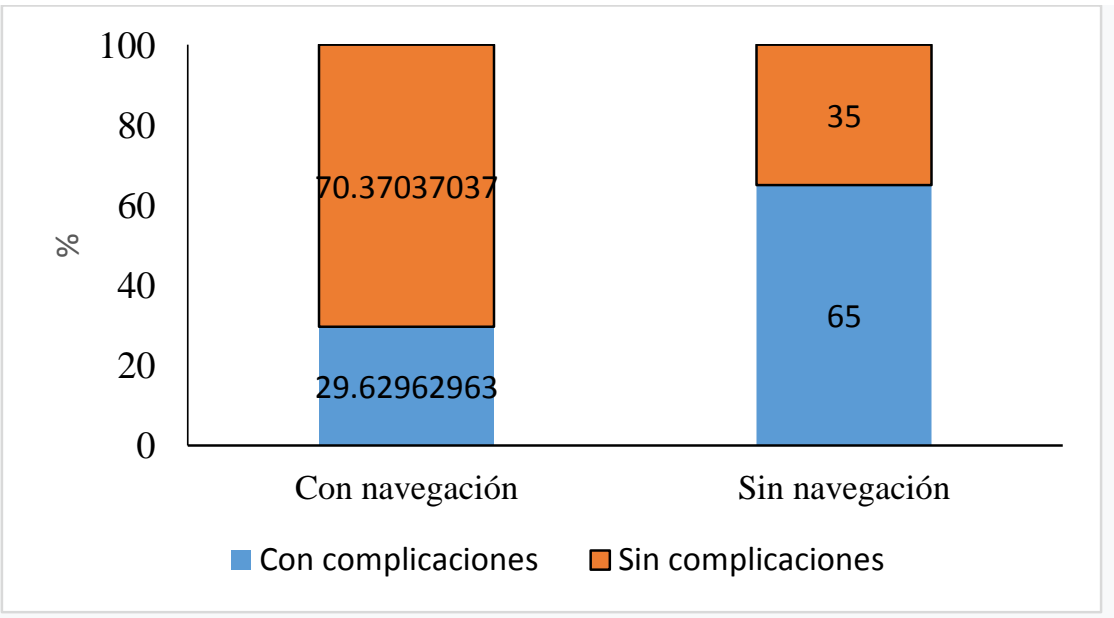

$\mathrm{p}=0,034$

Figure 4: Post-surgical complications with and without the use of neuronavigator.

\section{Discussion}

For the performance of any surgical procedure, a sense of threedimensional spatial perception of the anatomy of each patient is required; Intracranial location is a challenge for all neurosurgeons as they face each patient's own anatomical variations caused by the distortion caused by the underlying pathology, by the limited visual field product of the type of approach, the same location of the lesion, the anatomical complexity of the deeper areas of the brain, vascular relationships, proximity to eloquent areas, etc.

Diagnostic imaging methods based on a three-dimensional (3D) model, greatly facilitated the spatial location of all types of injuries, especially those that are more difficult to access and delimit by their location or distribution. The main link between imaging studies and the anatomy of each patient is due to the integration provided by navigation systems during surgery.

However, not all centers have neuronavigation equipment. In Argentina, in 2016, a series of 43 surgeries were reported without the use of neuronavigation in 10 years [13]. In our center, 20 patients were operated without a browser for computer processes related to the equipment.

In 2014, Valencia et al., Performed 10 callosotomies assisted with neuronavigation; In no case were intraoperative complications reported, concluding that callosotomy guided by frameless neuronavigation is an accurate and safe technique [11].

In Germany, in a series of 28 patients with lesional epilepsy, non-lesion and temporal epilepsy underwent surgery, 11 of them (39.3\%) used intraoperative MRI and neuronavigation; better results were obtained in the group that used neuronavigation as an aid in the development of surgery

[14].

If we compare the aforementioned studies with our research, in our series a greater number of surgeries were performed in less time (47 surgeries in 4 years), with a higher percentage of neuronavigator utilization (57.44\% vs. 39.3\% reported by Sommer) [14]. Amandeep Kumar, Sarat Chandra, et al. in 2014 they mention that the use of multimodal neuronavigation (with functional resonance and tractography) together with cerebral mapping with cortical stimulator for resection of lesions on eloquent cortical areas (motor, sensory, language) can reduce the risk of leaving the patient with permanent neurological sequelae and improving the percentage of resection of the affected area [15]. Björn Sommer, et al. In 2015, they describe how the use of intraoperative functional resonance and neuronavigation in ganglioglioma excision, (responsible for causing refractory epilepsy), improves the percentage of tumor resection, and therefore the number of seizures is significantly reduced [16]. In 2016, the same author Björn Sommer, et al, described how resection based on magnetoelectroencephalography with the use of neuronavigation and intraoperative MRI promises to be very useful in the complete resection of epileptogenic areas [17]. Karl Roessler et al. They report in 2016 that neuronavigation along with intraoperative MRI proved to be very useful in performing epilepsy surgery since it was associated with better resection of compromised cortical areas, with fewer complications and with better crisis control [18]. A systematic review carried out in Brazil in 2015 by Sovenso mentions that only one of the 28 studies analyzed reports that there was no significant difference in surgical time compared to the use or not of neuronavigation ( 239 vs 208 minutes). Four other studies analyzed only describe the mean surgical times with the use of neuronavigation (212 $\mathrm{min}, 213.3 \mathrm{~min}, 243 \mathrm{~min}$ and $213 \mathrm{~min}$ ) [19]. If we compare our study where the average surgical time was 198 minutes for the group that used neuronavigation, we see that the surgery time was shorter than the studies analyzed.

The Department of Neurosurgery of the School of Medicine of the University of Osaka Japan, Haruhiko Kishima et al. published in 2017 the utility of neuronavigation in the performance of selective amygdalohippocampectomy via infratemporal cortex, with excellent results in crisis control and memory preservation because the lobe is not damaged by the guidance of the neuronavigator during the surgical procedure temporary [20].

Regarding postoperative complications, in the Aberastury series of 43 patients operated without navigation, it reports 19 patients with new postsurgical neurological deficits of which 14 were permanent, 4 cases of post-surgical hydrocephalus, 7 cases of surgical site infections [13]. In our series, with the use of a neuronavigator, 9 patients presented neurological deficits, of which 8 were transient, 4 infections of the surgical site, no hydrocephalus was reported.

Roessler from Germany, in his study based on a series of patients operated with the use of neuronavigation does not report deaths, and a low percentage of hemiparesis and pneumonia [18].

In Germany, Delev and colleagues studied 40 patients in whom the use of neuronavigator was used in the planning of patients with apparently nonlesion epilepsy. It was shown that with the correct use of the neuronavigator tools, lesions were identified that were not initially seen on the MRI, and their use during the surgical procedure helped to perform more extensive resections, with better crisis control [21].

In summary, the refractory epilepsy in children is a debilitating condition, which impacts approximately 20 or $30 \%$ of the pediatric population that suffers from epilepsy. Epilepsy surgery improves not only the quality of life with crisis control, but it has been shown to improve cognition and behavior in patients undergoing surgery.

The challenge for the neurosurgeon is to translate the data from neuroimaging and neurophysiology recordings into a 3D space for surgical resection. The generation of a planned resection area clarifies the surgical 
strategy and gives the surgeon confidence that the proposed resection is complete [22].

\section{Conclusions}

We can conclude that since 2013 there has been a gradual increase in surgery for the control of refractory epilepsy with the use of neuronavigation as an aid in the development of surgeries; The use of this resource in pre and trans surgery is useful since it reduces the risk of the presence of trans and post-surgical complications associated with the procedure, decreases the surgical time, the amount of bleeding and even the days of hospitalization. Although the performance of epilepsy surgery with the use of neuronavigation has increased, it continues to be underutilized for the treatment of refractory epilepsy.

\section{Recommendations}

It is necessary to conduct training in the use of neuronavigation for the planning and development of surgeries for the control of refractory epilepsy. It is essential to establish protocols for diagnosis, evaluation and management of patients with refractory epilepsy, as well as to design a protocol for the use of neuronavigation in pre-surgical planning and its use during the development of epilepsy surgery. Surgery with the use of neuronavigation as a treatment for refractory epilepsy should be considered within the initial management to achieve better control of epileptic seizures.

\section{References}

1. Hauser WA, Annegers JF, Kurland LT. (1993) Incidence of epilepsy and unprovoked seizures in Rochester, Minnesota: 1935-1984. Epilepsia. junio de 34(3):453-468.

2. Sander JW. (2003) The epidemiology of epilepsy revisited. Curr Opin Neurol. abril de 16(2):165-170.

3. Kwan P, Sander JW. (2004) The natural history of epilepsy: an epidemiological view. J Neurol Neurosurg Psychiatry. octubre de 75(10):1376-1381.

4. Sánchez-Álvarez JC, Mauri-Llerda JA, Gil-Nagel A, CasasFernández C, Salas-Puig J, et al. (2012) Consensusrecommended diagnostic and therapeutic guidelines for drugresistant epilepsy in Spain (Consenso RATE-España). Neurol Barc Spain. octubre de 27(8):481-490.

5. López González FJ, Rodríguez Osorio X, Gil-Nagel Rein A, Carreño Martínez M, Serratosa Fernández J. et al. (2015) Drugresistant epilepsy: definition and treatment alternatives. Neurol Barc Spain. septiembre de 30(7):439-446.

6. Pittau F, Grouiller F, Spinelli L, Seeck M, Michel CM. (2014) The role of functional neuroimaging in pre-surgical epilepsy evaluation. Front Neurol. 5:31.

7. Sillanpää M, Shinnar S. (2011) Long-term mortality in childhood-onset epilepsy. N Engl J Med. 23 de diciembre de 2010;363(26):2522-2529.

8. de Tisi J, Bell GS, Peacock JL, McEvoy AW, Harkness WFJ, et al. (2011) The long-term outcome of adult epilepsy surgery, patterns of seizure remission, and relapse: a cohort study. Lancet Lond Engl. 15 de octubre de 378(9800):1388-1395.
9. Rosenow F, Lüders H. (2001) Presurgical evaluation of epilepsy. Brain J Neurol. septiembre de 124(Pt 9):1683-700.

10. Duncan JS, Winston GP, Koepp MJ, Ourselin S. (2016) Brain imaging in the assessment for epilepsy surgery. Lancet Neurol. abril de 15(4):420-433.

11. Valencia Calderón C, Castro Cevallos A, Calderón Valdiviezo A, Escobar Dávila R, Parra Rosales F, Quispe Alcocer J. (2016) [Neuronavigation in the surgical planning of callosotomy]. Neurocir Astur Spain. agosto de 27(4):186-193.

12. Gerard IJ, Kersten-Oertel M, Petrecca K, Sirhan D, Hall JA (2017) Brain shift in neuronavigation of brain tumors: A review. Med Image Anal. enero de 35:403-420.

13. Aberastury M, Comas B, García M, Besocke A, Ciraolo C, Agosta G, et al. (2016) Epilepsy surgery in children and adolescents: Report on 43 cases. Arch Argent Pediatr. 1 de octubre de 114(5):458-463.

14. Sommer B, Roessler K, Rampp S, Hamer HM, Blumcke I, et al.(2016) Magnetoencephalography-guided surgery in frontal lobe epilepsy using neuronavigation and intraoperative MR imaging. Epilepsy Res. octubre de, 126:26-36.

15. Kumar A, Chandra PS, Sharma BS, Garg A, Rath GK. et al. (2014) The role of neuronavigation-guided functional MRI and diffusion tensor tractography along with cortical stimulation in patients with eloquent cortex lesions. Br J Neurosurg. abril de 28(2):226-233.

16. Sommer B, Wimmer C, Coras R, Blumcke I, Lorber B. et al. (2015) Resection of cerebral gangliogliomas causing drugresistant epilepsy: short- and long-term outcomes using intraoperative MRI and neuronavigation. Neurosurg Focus. enero de, 38(1):E5.

17. Sommer B, Roessler K, Rampp S, Hamer HM, Blumcke I, Stefan H, et al. (2016) Magnetoencephalography-guided surgery in frontal lobe epilepsy using neuronavigation and intraoperative MR imaging. Epilepsy Res. octubre de, 126:26-36.

18. Roessler K, Sommer B, Merkel A, Rampp S, Gollwitzer S, Hamer HM, et al. (2016) A Frameless Stereotactic Implantation Technique for Depth Electrodes in Refractory Epilepsy Using Intraoperative Magnetic Resonance Imaging. World Neurosurg. octubre de, 94:206-210.

19. Sonvenso DK, Itikawa EN, Santos MV, Santos LA, Trevisan AC, Bianchin MM, et al. (2015) Systematic review of the efficacy in seizure control and safety of neuronavigation in epilepsy surgery: The need for well-designed prospective studies. Seizure. septiembre de 31:99-107.

20. Kishima H, Kato A, Oshino S, Tani N, Maruo T, Khoo HM, et al. (2017) Navigation-assisted trans-inferotemporal cortex selective amygdalohippocampectomy for mesial temporal lobe epilepsy; preserving the temporal stem. Neurol Res. marzo de, 39(3):223-30.

21. Delev D, Quesada CM, Grote A, Boström JP, Elger C, Vatter H, et al. (2017) A multimodal concept for invasive diagnostics and surgery based on neuronavigated voxel-based morphometric MRI postprocessing data in previously nonlesional epilepsy. $\mathbf{J}$ Neurosurg. 16 de junio de, 1-9.

22. Nowell M, Sparks R, Zombori G, Miserocchi A, Rodionov R, Diehl B, et al. (2017) Resection planning in extratemporal epilepsy surgery using 3D multimodality imaging and intraoperative MRI. Br J Neurosurg. 31(4): 468-470. 